\title{
EFFECT OF ARTIFICIAL ACID RAIN ON ALUMINIUM TANNED LEATHER
}

\section{INFLUENTA PLOII ACIDE ARTIFICIALE ASUPRA PIELII TĂBĂCITE CU ALUMINIU}

\author{
Yanping GAO ${ }^{1,2}$, Shuang YANG ${ }^{1,2}$, Xiaoyun JIAN ${ }^{1,2}$, Lucreţia MIU ${ }^{3}$, Carmen GAIDĂU ${ }^{3}$, Wuyong CHEN $^{1,2^{*}}$ \\ ${ }^{1}$ Key Laboratory of Leather Chemistry and Engineering of the Ministry of Education, Sichuan University, Chengdu, 610065, P. R. China \\ ${ }^{2}$ National Engineering Laboratory for Clean Technology of Leather Manufacture, Sichuan University, Chengdu 610065, P. R. China \\ ${ }^{3}$ INCDTP - Division: Leather and Footwear Research Institute, 93 Ion Minulescu St., sector 3, 031215, Bucharest, Romania
}

\begin{abstract}
EFFECT OF ARTIFICIAL ACID RAIN ON ALUMINIUM TANNED LEATHER
ABSTRACT. This article aims at studying the influence of gaseous pollutants on aluminium tanned leather. Artificial acid rain was used to soak the aluminium tanned collagen materials for $0,4,8,12$ and 16 days, then some analytical techniques were used to evaluate the changes of leather during the aging process. Micro hot table (MHT), differential scanning calorimetry (DSC) and thermogravimetry (TG) were used to evaluate the influence of artificial acid rain on thermal behaviors. Scanning electron microscope(SEM) was used to investigate the changes of fibrous morphology during aging process. Fourier transform infrared spectroscopy (FT-IR) was used to investigate the changes of structural property after artificial acid rain treatment. With the extension of aging time, thermal stability and heat resistance were gradually reduced, including shrinkage temperature, thermal denaturing temperature, associated enthalpy, weight loss and temperature for maximum decomposition rate. SEM images showed that the collagen fibers were greatly damaged, the gaps among fibres were disappeared and the fibre bundles were randomly ruptured with the aging time. The FT-IR results showed that, during the aging process, amide A band was moved to higher numbers, amide I and amide II band of collagen were gradually shifted to lower numbers. Consequently, the structure and performance of aluminium tanned leather were damaged by artificial acid rain and the longer the aging time is, the greater the damage is.

KEY WORDS: leather making; aluminium tanned leather; artificial acid rain; aging
\end{abstract}

\section{INFLUENTA PLOII ACIDE ARTIFICIALE ASUPRA PIELII TABACITE CU ALUMINIU}

REZUMAT. Obiectivul acestui articol este acela de a studia influenta poluantilor gazosi asupra pielii tabacite cu aluminiu. S-a utilizat ploaia acida artificiala pentru a înmuia materialul colagenic tabacit cu aluminiu timp de $0,4,8,12$ si 16 zile, apoi s-au efectuat anumite analize pentru a evalua modificarile pielii în timpul procesului de îmbatrânire. S-au utilizat tehnicile Micro Hot Table (MHT), calorimetria cu scanare diferentiala (DSC) si termogravimetria (TG) pentru a evalua influenta ploii acide artificiale asupra comportamentului termic. Microscopia electronica de baleiaj (SEM) a fost utilizata pentru a investiga modificarile morfologiei fibroase în timpul procesului de îmbatrânire. Spectroscopia în infrarosu cu transformata Fourier (FT-IR) a fost utilizata pentru a investiga modificarile proprietatilor structurale dupa tratamentul cu ploaie acida artificiala. Odata cu prelungirea timpului de îmbatrânire, stabilitatea termica si rezistenta la caldura s-au redus treptat, inclusiv temperatura de contractie, temperatura de denaturare termica, entalpia asociata, pierderea în greutate si temperatura ratei maxime de descompunere. Imaginile SEM au aratat ca fibrele de colagen au fost foarte deteriorate, golurile dintre fibre au disparut, iar fasciculele de fibre s-au rupt în mod aleatoriu, odata cu prelungirea timpului de îmbatrânire. Rezultatele analizei FT-IR au aratat ca, în timpul procesului de îmbatrânire, banda amida A s-a deplasat la valori mai mari, benzile amida I si amida II ale colagenului s-au deplasat treptat spre valori mai mici. În consecinta, structura si performanta pielii tabacite cu aluminiu au fost afectate de ploaia acida artificiala si cu cât este mai mare timpul de îmbatrânire, cu atât este deteriorarea mai mare.

CUVINTE CHEIE: fabricarea pielii; piele tabacita cu aluminiu; ploaie acida artificiala; îmbatrânire

\section{L'EFFET DE LA PLUIE ACIDE ARTIFICIELLE SUR LE CUIR TANNÉ À L'ALUMINUM}

RÉSUMÉ. Cet article vise à étudier l'influence des polluants gazeux sur le cuir tanné à l'aluminum. On a utilisé la pluie acide artificielle pour tremper le matériau de collagène tanné à l'aluminum pendant 0, 4, 8, 12 et 16 jours, puis quelques techniques d'analyse ont été utilisées pour évaluer les changements du cuir pendant le processus de vieillissement. On a utilisé des techniques telles que Micro Hot Table (MHT), la calorimétrie différentielle à balayage (DSC) et la thermogravimétrie (TG) pour évaluer l'influence de la pluie acide artificielle sur le comportement thermique. La microscopie électronique à balayage (MEB) a été utilisée pour étudier les changements de morphologie fibreuse pendant le processus de vieillissement. La spectroscopie infrarouge à transformée de Fourier (FT-IR) a été utilisée pour étudier les changements des propriétés structurelles après le traitement de la pluie acide artificielle. Avec l'extension du temps de vieillissement, la stabilité thermique et la résistance à la chaleur ont été progressivement réduites, y compris la température de rétraction, la température de dénaturation thermique, l'enthalpie associée, la perte de poids et la température pour le taux maximal de décomposition. Les images au MEB ont montré que les fibres de collagène sont fortement endommagées, les écarts entre les fibres ont disparu et les faisceaux de fibres sont rompues de façon aléatoire avec l'extension du temps de vieillissement. Les résultats FT-IR ont montré que, au cours du processus de vieillissement, la bande amide A a été déplacée à un plus grand nombre, les bandes amide I et amide II de collagène ont été progressivement déplacées à un nombre plus réduit. En conséquence, la structure et la performance du cuir tanné à l'aluminum ont été endommagées par la pluie acide artificielle et le plus long le temps de vieillissement, le plus grand le dommage.

MOTS CLÉS: fabrication du cuir; cuir tanné à l'aluminum; pluie acide artificielle; vieillissement

" Correspondence to: Wuyong CHEN, Key Laboratory of Leather Chemistry and Engineering of the Ministry of Education, Sichuan University, Chengdu, 610065, P. R. China, email: wuyong.chen@163.com, Tel: (+86)028-85405237, Fax:(+86)028-85405237 


\section{INTRODUCTION}

Aluminium tanning is one of the oldest tanning methods which was widely used in upper leather, garment leather, glove leather and fur leather before chrome tanning method. Therefore, large proportion of collagen-based cultural relics was made of aluminium tanned leather, for example the imperial crown dating back to 3000 BC made of aluminium tanned leather which discovered in the grave of King Sumerian in the Euphrates [1]. In addition, according to the historical records, alum was used to leather making from Warring States Period in China. A pair of cattle hide boot was made in two thousand years ago which preserved in Beijing Museum was aluminium tanned leather product [2].

Many objects made of aluminium tanned leather are valuable treasures due to the history they represent, and their preservation challenges museum custodians and private collectors alike [3]. A major problem in the preservation of these relics is the damage caused by the effect of temperature and relative humidity, excessive light and air pollution. The last one is considered as one of the main factors accelerating the degradation and damage of the cultural heritage, especially such collagen-based cultural heritage (paper, parchment and leather etc.) [4].

On the one hand, in order to protect historical objects, new types of leather were studied to use for the restoration of historical objects $[5,6]$. On the other hand, the effect of pollutant gases on collagen-based materials were studied. It has been already demonstrated that the corrosion of leather was caused by $\mathrm{SO}_{2}$ and the leather may become powder by rubbing if the damage was severe [7]. Besides, environmental chemical pollutants for example $\mathrm{NO}_{2}$ and $\mathrm{SO}_{2}$ etc. had a great impact on the thermal degradation of collagenbased materials $[8,9]$. Moreover, in previous paper, assessment of the air quality in museums was studied for better preservation of cultural heritages [4]. However, the studies of synthetic research techniques on the effect of gaseous pollutants on aluminium tanned leather are still rarely reported.

The components of atmospheric air which belong to the most common pollutants are sulphur dioxide $\left(\mathrm{SO}_{2}\right)$, nitric oxide $\left(\mathrm{NO}_{x}\right)$ and air suspended particulate

\section{INTRODUCERE}

Tăbăcirea cu aluminiu este una dintre cele mai vechi metode de tăbăcire, utilizată pe scară largă pentru feţe de încălţăminte, îmbrăcăminte, mănuşi şi blănuri înainte de metoda de tăbăcire în crom. Prin urmare, o mare parte dintre relicvele culturale din materiale pe bază de colagen au fost făcute din piele tăbăcită cu aluminiu, de exemplu coroana imperială din piele tăbăcită cu aluminiu, datând de la 3000 î.Hr., care a fost descoperită în mormântul unui rege sumerian din regiunea Eufratului [1]. În plus, conform înregistrărilor istorice, alaunul a fost folosit pentru procesul de fabricare a pielii în China din Perioada Statelor Combatante. $O$ pereche de cizme din piele de bovine de acum două mii de ani, păstrată la Muzeul din Beijing, a fost fabricată din piele tăbăcită cu aluminiu [2].

Multe obiecte din piele tăbăcită cu aluminiu sunt comori valoroase datorită istoriei pe care o reprezintă, iar conservarea lor constituie o provocare pentru muzeografi şi colecţionari privaţi deopotrivă [3]. O problemă majoră legată de conservarea acestor relicve o reprezintă deteriorările cauzate de temperatură şi umiditate relativă, lumină excesivă şi poluarea aerului. Cea din urmă este considerată unul dintre principalii factori care accelerează degradarea şi deteriorarea patrimoniului cultural, în special obiectele pe bază de colagen (hârtie, pergament, piele etc.) [4].

Pe de o parte, pentru a proteja obiectele istorice, s-au studiat noi tipuri de piele în vederea utilizării la restaurarea obiectelor istorice $[5,6]$. Pe de altă parte, sa studiat influenţa gazelor poluante asupra materialelor pe bază de colagen. S-a demonstrat deja că $\mathrm{SO}_{2}$ cauzează coroziunea pielii, iar aceasta se poate transforma în praf prin frecare dacă gradul de deteriorare este înalt [7]. În plus, poluanţii chimici care

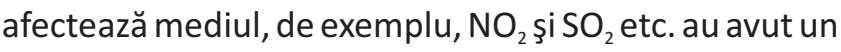
impact mare asupra degradării termice a materialelor pe bază de colagen $[8,9]$. Mai mult, în lucrări anterioare $\mathrm{s}$-a evaluat calitatea aerului în muzee pentru o mai bună conservare a patrimoniului cultural [4]. Cu toate acestea, studiile care sintetizează tehnicile de cercetare privind efectul poluanţilor gazoşi asupra pielii tăbăcite cu aluminiu sunt încă foarte rar raportate.

Componentele aerului atmosferic care aparţin celor mai frecvenţi poluanţi sunt dioxidul de sulf $\left(\mathrm{SO}_{2}\right)$, monoxidul de azot $\left(\mathrm{NO}_{x}\right)$ şi particulele în suspensie 
matter (PM). With the presence of humidity, $\mathrm{NO}_{2}$ can be converted into nitric acid $\left(\mathrm{HNO}_{3}\right)$ and $\mathrm{SO}_{2}$, if also oxidants are available into sulphuric acid $\left(\mathrm{H}_{2} \mathrm{SO}_{4}\right)$. Acidic air pollution leads to the hydrolytic degradation of collagen-based materials meanwhile causing a significant damage to the artifacts.

In this research, the artificial acid rain was used to simulate the effect of air pollutants on aluminium tanned leather. The aluminium tanned leather samples were soaked in the artificial acid rain for 0,4 , 8,12 , and 16 days, at a $50^{\circ} \mathrm{C}$ and $100 \%$ relative humidity condition. After aging test, DSC, TG and MHT were used to evaluate the variation of their thermal behaviors; FT-IR and SEM were used to investigate the changes of structural and histological properties [10, 11].

This study aims at providing valuable references for storage of aluminium tanned leather cultural relics. Moreover, it may form the basis for improving the methods of artificial aging.

\section{EXPERIMENTAL METHODOLOGY}

\section{Materials}

Limed hides, Sichuan China; Chemicals used for tanning process were all industrial grade; Chemicals used for analysis were all research grade.

\section{Preparation of Aluminium Tanned Leather}

Aluminium tanned leather was made by simulating the traditional tannage, so deliming liquor in the modern leather making process was adjusted. Formic acid, acetic acid and lactic acid were used in delime process to simulate the outcome after wheat bran fermentation in ancient leather making method [12]. Leather was tanned by alum in a rectangular basin and stirred by labor, the aluminium leather was made after 2 weeks. The technology of aluminium tanned leather is shown in Table 1.
(PM). În prezenţa umidităţii, $\mathrm{NO}_{2}$ poate fi transformat în acid azotic $\left(\mathrm{HNO}_{3}\right)$ şi $\mathrm{SO}_{2}$, dacă există şi oxidanţi în acidul sulfuric $\left(\mathrm{H}_{2} \mathrm{SO}_{4}\right)$. Poluarea acidă a aerului duce la degradarea hidrolitică a materialelor pe bază de colagen, provocând în acelaşi timp deteriorări semnificative artefactelor.

În această lucrare de cercetare, s-a utilizat ploaia acidă artificială pentru a simula efectul poluanţilor atmosferici asupra pielii tăbăcite cu aluminiu. Probele de piele tăbăcite cu aluminiu au fost înmuiate în ploaie acidă artificială timp de $0,4,8,12$ şi 16 zile, la $50^{\circ} \mathrm{C}$ şi $100 \%$ umiditate relativă. După testul de îmbătrânire, sau utilizat tehnicile DSC, TG şi MHT pentru a evalua variaţia comportamentului termic al probelor; FT-IR şi SEM au fost utilizate pentru a investiga modificările proprietăţilor structurale şi histologice [10,11].

Acest studiu îşi propune să ofere referinţe valoroase pentru depozitarea relicvelor culturale din piele tăbăcită cu aluminiu. Mai mult decât atât, acesta poate forma o bază pentru îmbunătăţirea metodelor de îmbătrânire artificială.

\section{METODOLOGIA EXPERIMENTALĂ}

\section{Materiale}

Piei cenuşărite, Sichuan China; toate substanţele chimice utilizate în procesul de tăbăcire au fost de calitate industrială; toate substanţele chimice utilizate pentru analiză au fost de calitate analitică.

\section{Prelucrarea pielii tăbăcite cu aluminiu}

Pielea tăbăcită cu aluminiu a fost prelucrată simulând procesul tradiţional de tăbăcire, aşadar s-a ajustat flota de la decalcificare din cadrul procesului modern de fabricare a pielii. În procesul de decalcificare s-au utilizat acid formic, acid acetic şi acid lactic pentru a simula rezultatul obţinut după fermentarea tărâţelor de grâu utilizate în metoda veche de fabricare a pielii [12]. Pielea a fost tăbăcită cu alaun într-un vas dreptunghiular şi agitat manual, pielea tăbăcindu-se cu aluminiu după 2 săptămâni. Tehnologia de tăbăcire cu aluminiu este prezentată în Tabelul 1. 
Table 1: Technology of aluminium tanned leather

Tabelul 1: Tehnologia de tăbăcire a pielii cu aluminiu

\begin{tabular}{|c|c|c|c|c|c|}
\hline $\begin{array}{l}\text { Process } \\
\text { Proces }\end{array}$ & $\%$ & $\begin{array}{l}\text { Chemical } \\
\text { Substanţă chimică }\end{array}$ & $\mathrm{T} /{ }^{\circ} \mathrm{C}$ & $\mathrm{t} / \mathrm{min}$ & $\begin{array}{l}\text { Comment } \\
\text { Observaţii }\end{array}$ \\
\hline $\begin{array}{l}\text { Weigh } \\
\text { Cântărire }\end{array}$ & & & & & $\begin{array}{l}\text { As the standard dosage } \\
\text { Conform standardului de dozare }\end{array}$ \\
\hline $\begin{array}{l}\text { Wash } \\
\text { Spălare }\end{array}$ & 300 & $\begin{array}{l}\text { Water } \\
\text { Apă }\end{array}$ & 25 & 10 & \\
\hline \multirow{4}{*}{$\begin{array}{l}\text { Delime } \\
\text { Decalcificare }\end{array}$} & 300 & $\begin{array}{l}\text { Water } \\
\text { Apă }\end{array}$ & 35 & & $12 \mathrm{rpm}$ \\
\hline & 0.02 & $\begin{array}{l}\text { Formic acid } \\
\text { Acid formic }\end{array}$ & & 180 & \\
\hline & 0.04 & $\begin{array}{l}\text { Acetic acid } \\
\text { Acid acetic }\end{array}$ & & & \\
\hline & 0.30 & $\begin{array}{l}\text { Lactic acid } \\
\text { Acid lactic }\end{array}$ & & & \\
\hline $\begin{array}{l}\text { Wash } \\
\text { Spălare }\end{array}$ & 300 & $\begin{array}{l}\text { Water } \\
\text { Apă }\end{array}$ & 30 & $10 \times 2$ & \\
\hline \multirow{4}{*}{$\begin{array}{l}\text { Tannage } \\
\text { Tăbăcire }\end{array}$} & 50 & $\begin{array}{l}\text { Water } \\
\text { Apă }\end{array}$ & 25 & & \\
\hline & 4.5 & $\mathrm{KAI}\left(\mathrm{SO}_{4}\right)_{2} \cdot 12 \mathrm{H}_{2} \mathrm{O}$ & & $1440 \times 14$ & \multirow{2}{*}{$\begin{array}{l}\mathrm{KAI}\left(\mathrm{SO}_{4}\right)_{2} \cdot 12 \mathrm{H}_{2} \mathrm{O} \text { and } \mathrm{NaCl} \text { are } \\
\text { dissolved in water in advance. Stir } 10 \\
\text { min, run } 5 \text { mins/h, overnight. } \\
\mathrm{KAI}\left(\mathrm{SO}_{4}\right)_{2} \cdot 12 \mathrm{H}_{2} \mathrm{O} \text { şi NaCl se dizolvă în } \\
\text { apă în prealabil. Se agită timp de } 10 \\
\text { min, se pune în funcţiune timp de } 5 \\
\text { min/h, se lasă peste noapte. }\end{array}$} \\
\hline & 1.5 & $\mathrm{NaCl}$ & & & \\
\hline & 1.75 & $\mathrm{NaHCO}_{3}$ & & & pH 3.99 \\
\hline $\begin{array}{l}\text { Wash } \\
\text { Spălare }\end{array}$ & 300 & & 25 & $10 \times 2$ & \\
\hline $\begin{array}{l}\text { Dry } \\
\text { Uscare }\end{array}$ & & & & & $\begin{array}{c}\text { Horse up, lay down, flat dry } \\
\text { Întindere pe capră, scoatere, uscare }\end{array}$ \\
\hline
\end{tabular}

\section{Preparation of Artificial Acid Rain}

The artificial acid rain was prepared as follows: 0.04 $\mathrm{mL}$ sulphuric acid, $0.06 \mathrm{~mL}$ hydrochloric acid and $0.02 \mathrm{~mL}$ ammonium hydroxide were dissolved in $80 \mathrm{~mL}$ distilled water, then $0.0296 \mathrm{~g} \mathrm{Ca}(\mathrm{OH})_{2}$ was added in the mixture. Then, the solution was diluted to $2 \mathrm{~L}$ with distilled water. The $\mathrm{pH}$ of the artificial rain was 3.25 [13].

\section{Leather Aging Process}

Aluminium tanned leather was cut into pieces, and then these pieces were soaked in the artificial acid rain (1:20, mass of leather: mass of acid rain) and

\section{Prepararea ploii acide artificiale}

Ploaia artificială a fost preparată după cum urmează: s-au dizolvat 0,04 $\mathrm{ml}$ acid sulfuric, $0,06 \mathrm{ml}$ acid clorhidric şi $0,02 \mathrm{ml}$ hidroxid de amoniu în $80 \mathrm{ml}$ apă distilată, apoi s-a adăugat $0,0296 \mathrm{~g} \mathrm{Ca}(\mathrm{OH})_{2}$ în amestec. Soluţia a fost apoi diluată cu apă distilată până la 2 L. pHul soluţiei de ploaie acidă artificială a fost 3,25 [13].

\section{Procesul de îmbătrânire a pielii}

Pielea tăbăcită cu aluminiu a fost tăiată în bucăţi care au fost înmuiate în soluţia de ploaie acidă (1:20, masă piele:masă ploaie acidă) şi menţinute într-o 
kept in a constant temperature and humidity chamber (LHS-300(F), Shanghai FEIYUE experiment instrument CO.,LTD.) at $50^{\circ} \mathrm{C}$ and $100 \%$ relative humidity $(\mathrm{RH})$ for $0,4,8,12$, and 16 days. After natural drying 48 hours, treated samples were placed in a $65 \%$ relative humidity desiccator for over a week until constant weight.

\section{MHT Analysis}

MHT measurements were performed with an easyto-use equipment composed of a stereo microscope (SBM-20TF China) and a hot table A WT2000 (China) equipped with a temperature processor and a homemade software for temperature regulation and data collection. Magnification used was $\times 40$.

A sample (1-2 mg) of fibers from the corium part of the samples was divided into triplicates. Each subsample was washed with distilled water for $10 \mathrm{~min}$, placed on microscope slide with a concavity and covered with distilled water and a microscope slip, and more than two fibers should be observed in the sight. The slide was placed on the hot table and heated at a rate of $2^{\circ} \mathrm{C} \mathrm{min}^{-1}$. Ts was recorded when more than two fibers were shrinked simultaneously and continuously. The final results were the average of three tests [14].

\section{DSC Analysis}

DSC measurements on leather samples were performed in the temperature range from 20 to $250^{\circ} \mathrm{C}$, at $10^{\circ} \mathrm{C} \mathrm{min}^{-1}$ heating rate in dry nitrogen flow $(20 \mathrm{~mL}$ $\mathrm{min}^{-1}$ ) in sealed aluminium crucibles. Samples were analyzed in a dry condition after a few days' storage in a controlled environment (approximately $20^{\circ} \mathrm{C}$ and $65 \%$ $\mathrm{RH}$ ). The sample masses were 3 to $5 \mathrm{mg}$. Measurements were made with a NETZSCH DSC PC200 calorimeter (Germany).

\section{TG Analysis}

TG and DTG were simultaneously recorded with a NETZSCH TG 209 F1 thermogravimetric analyzer (Germany) from 40 to $600^{\circ} \mathrm{C}$, at $10^{\circ} \mathrm{C} \mathrm{min}^{-1}$ heating rate. Measurements were made in dry nitrogen flow $(20 \mathrm{~mL}$ $\min ^{-1}$ ) using $\mathrm{Al}_{2} \mathrm{O}_{3}$ crucibles. Samples were analyzed in a dry condition after a few days' storage in a controlled environment (approximately $20^{\circ} \mathrm{C}$ and $65 \% \mathrm{RH}$ ). The sample masses were 3 to $5 \mathrm{mg}$. cameră cu temperatură şi umiditate constante (LHS300(F), Shanghai FEIYUE experiment instrument CO., LTD.) la $50^{\circ} \mathrm{C}$ şi $100 \%$ umiditate relativă (RH) timp de 0 , 4, 8, 12 şi 16 zile. După uscare naturală timp de 48 de ore, probele tratate au fost puse într-un exsicator cu $65 \%$ umiditate relativă timp de o săptămână până ce au ajuns la o greutate constantă.

\section{Analiza MHT}

Măsurătorile MHT s-au efectuat cu un echipament uşor de utilizat, compus dintr-un microscop stereo (SBM20TF China) şi o microplacă încălzită A WT2000 (China) cuplată cu un procesor de temperatură şi un software propriu pentru reglarea temperaturii şi colectarea datelor. Mărirea utilizată a fost X40.

Un eşantion (1-2 mg) de fibre din partea cu dermă a probelor a fost împărţit în trei. Fiecare sub-eşantion a fost spălat cu apă distilată timp de $10 \mathrm{~min}$, plasat pe lama concavă de microscop şi acoperit cu apă distilată şi o lamelă de acoperire, putându-se observa cu ochiul liber mai mult de două fibre. Lama a fost plasată pe microplacă şi s-a încălzit cu o viteză de $2^{\circ} \mathrm{C} \mathrm{min}^{-1}$. S-a înregistrat $T_{\mathrm{s}}$ atunci când s-au contractat mai mult de două fibre simultan şi continuu. Rezultatele finale au reprezentat media a trei încercări [14].

\section{Analiza DSC}

Măsurătorile DSC s-au efectuat pe probele de piele în intervalul de temperatură $20-250^{\circ} \mathrm{C}$, la viteza de încălzire de $10^{\circ} \mathrm{C} \mathrm{min}^{-1}$ în curent de azot anhidru $(20 \mathrm{ml}$ $\mathrm{min}^{-1}$ ) în creuzete de aluminiu sigilate. Probele au fost analizate în stare uscată, după o depozitare de câteva zile într-un mediu controlat (aproximativ $20^{\circ} \mathrm{C}$ şi $65 \%$ $\mathrm{RH}$ ). Masele probelor au fost de 3 până la $5 \mathrm{mg}$. Măsurătorile au fost realizate cu un calorimetru NETZSCH DSC PC200 (Germania).

\section{Analiza TG}

Valorile TG şi DTG au fost înregistrate simultan cu un analizor termogravimetric NETZSCH TG 209 F1 (Germania) în intervalul $40-600^{\circ} \mathrm{C}$, la viteza de încălzire de $10^{\circ} \mathrm{C} \mathrm{min}^{-1}$. Măsurătorile au fost efectuate în curent de azot anhidru $\left(20 \mathrm{ml} \mathrm{min}^{-1}\right)$, folosind creuzete $\operatorname{din} \mathrm{Al}_{2} \mathrm{O}_{3}$. Probele au fost analizate în stare uscată, după o depozitare de câteva zile într-un mediu controlat (aproximativ $20^{\circ} \mathrm{C}$ şi $65 \% \mathrm{RH}$ ). Masele probelor au fost de la 3 până la $5 \mathrm{mg}$. 


\section{SEM Analysis}

A scanning electron microscope, JSM-5900LV, was used for the investigation of the surface morphology of the leather. All samples were photographed by SEM at the Scanning Electron Microscope Laboratory, the Central Laboratory Unit, Sichuan University, China. The samples were sliced into $30 \mu \mathrm{m}$ pieces by freezing microtome (CM1950, Leica, German), and then the 5 $\mathrm{nm}$ fine gold coating was plated on the slices. The cross sections were observed at 50 times magnification. In order to observe the changes of collagen fibres, the magnification times were 1000 .

\section{FT-IR Analysis}

FTIR spectra in the $4000-400 \mathrm{~cm}^{-1}$ were recorded with a Nicolet 10 FT-IR spectrophotometer (American Thermo Scientific Corporation) equipped with a DLATGS detector and a KBr beam splitter. Samples were prepared by grinding about $1 \mathrm{mg}$ leather with $100 \mathrm{mg}$ $\mathrm{KBr}$ and pressing the mixture into very thin disks and were scanned for 32 times. The maximum resolution of measurements was $1 \mathrm{~cm}^{-1}$.

\section{Analiza SEM}

S-a utilizat un microscop electronic cu baleiaj, JSM-5900LV, pentru investigarea morfologiei suprafeţei pielii. Toate probele au fost fotografiate în Laboratorul de Microscopie Electronică cu Baleiaj, Laboratorul Central, Universitatea Sichuan, China. Probele au fost tăiate în bucăţi de $30 \mu \mathrm{m}$ prin congelare cu microtom (CM1950, Leica, Germania), apoi au fost acoperite cu un strat de aur de $5 \mathrm{~nm}$. Secţiunile transversale au fost observate la o mărire de 50 de ori. Pentru a observa modificările fibrelor de colagen, s-a mărit imaginea de 1000 de ori.

\section{Analiza FT-IR}

Spectrele FTIR în regiunea $4000-400 \mathrm{~cm}^{-1}$ au fost înregistrate $\mathrm{cu}$ un spectrofotometru Nicolet 10 FT-IR (American Thermo Scientific Corporation) echipat $\mathrm{cu}$ un detector DLATGS şi un separator de fascicule $\mathrm{KBr}$. Probele au fost preparate prin măcinarea a aproximativ $1 \mathrm{mg}$ de piele cu $100 \mathrm{mg} \mathrm{KBr}$ şi presarea amestecului în discuri foarte subţiri, care au fost scanate de 32 de ori. Rezoluţia maximă a măsurătorilor a fost de $1 \mathrm{~cm}^{-1}$.

\section{RESULTS AND DISCUSSION}

\section{REZULTATE ŞI DISCUTII}

\section{Thermal Analysis}

Shrinkage Temperature

\section{Analiza termică}

Temperatura de contracţie

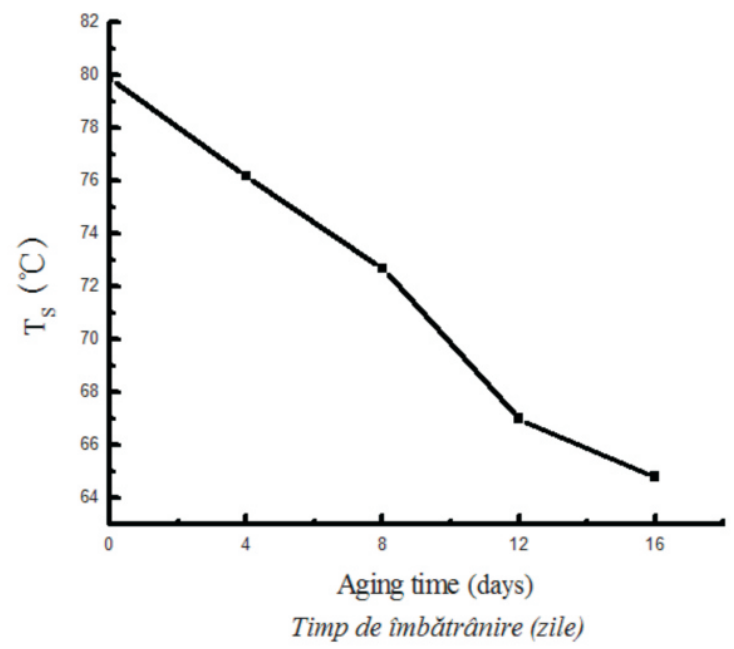

Figure 1. Shrinkage temperature of aluminium tanned leather after artificial acid rain treatment

Figura 1. Temperatura de contracţie a pielii tăbăcite cu aluminiu după tratamentul cu ploaie acidă artificială 
Figure 1 shows the $T_{\mathrm{s}}$ of aluminium tanned leather as a function of aging days. The $T_{\mathrm{s}}$ of matched sample is $79.9^{\circ} \mathrm{C}$ and then reduces to $64.9^{\circ} \mathrm{C}$ after aging 16 days, the $\Delta T$ is 15.1. The $T_{\mathrm{s}}$ firstly drops almost in a straight line from 4 to 12 days, and then shows little change from 12 to 16 days. The shrinkage temperature of leather is a valuable indicator of the hydrothermal stability of collagen, the decreasing $T_{\mathrm{s}}$ represents the decreased hydrothermal stability [14].

\section{DSC Properties}

Figura 1 prezintă $T_{\mathrm{s}}$ a pielii tăbăcite cu aluminiu pe parcursul perioadei de îmbătrânire. $T_{\mathrm{s}}$ a probei este iniţial $79,9^{\circ} \mathrm{C}$ şi scade la $64,9^{\circ} \mathrm{C}$ după 16 zile de îmbătrânire, $\Delta T$ este 15,1 . La început, $T_{\mathrm{s}}$ scade aproape în linie dreaptă de la 4 la 12 zile, apoi, de la 12 la 16 zile, nu prezintă modificări semnificative. Temperatura de contracţie a pielii este un indicator valoros al stabilităţii hidrotermice a colagenului, $T_{s}$ în scădere reprezentând scăderea stabilităţii hidrotermice [14].

\section{Proprietăţi DSC}

Table 2: The denaturation temperature and the associated enthalpy of aluminium tanned leather after artificial acid rain treatment

Tabelul 2: Temperatura de denaturare şi entalpia asociată pielii tăbăcite cu aluminiu după tratamentul cu ploaie acidă artificială

\begin{tabular}{c|c|c|c|}
$\begin{array}{c}\text { Aging time (days) } \\
\text { Timp îmbătrânire (zile) }\end{array}$ & $T_{\mathrm{d}}\left({ }^{\circ} \mathrm{C}\right)$ & $\Delta T_{\mathrm{d}}\left({ }^{\circ} \mathrm{C}\right)$ & $\Delta_{\mathrm{d}} H\left(\mathrm{~J} \mathrm{~kg}^{-1}\right)$ \\
\hline 0 & 90.5 & 0 & 361.8 \\
\hline 4 & 81.2 & 9.3 & 281.4 \\
\hline 8 & 79.8 & 10.7 & 246.4 \\
\hline 12 & 75.5 & 15.0 & 241.9 \\
\hline 16 & 74.8 & 15.7 & 224.3 \\
\hline
\end{tabular}

DSC analysis of samples allows evaluating the denaturation temperature $\left(T_{d}\right)$ of the samples. $\Delta_{d} H$ is peak area of the DSC curves and represents associated enthalpy during heating process. Both $\mathrm{Td}$ and $\Delta_{\mathrm{d}} H$ (Table 1) decrease with the aging time, $T_{\mathrm{d}}$ reduces from 90.5 to $74.8^{\circ} \mathrm{C}$ and $\Delta_{\mathrm{d}} H$ drops from 361.8 to $224.3 \mathrm{~J} \mathrm{~kg}^{-1}$. According to previous DSC studies, lower $T_{\mathrm{d}}$ and $\Delta_{\mathrm{d}} H$ values reflect decreased the thermal stability of collagen caused by its progressive deterioration [8].
Analiza DSC a probelor permite evaluarea temperaturii de denaturare $\left(T_{\mathrm{d}}\right)$ a probelor. $\Delta_{\mathrm{d}} H$ este zona de vârf a curbelor DSC şi reprezintă entalpia asociată în timpul procesului de încălzire. Atât $T_{\mathrm{d}}$ cât şi $\Delta_{\mathrm{d}} H$ (Tabelul 1) scad odată cu timpul de îmbătrânire, $T_{\mathrm{d}}$ scade de la $90,5 \mathrm{la}$ $74,8^{\circ} \mathrm{C}$, iar $\Delta_{\mathrm{d}} H$ scade de la 361,8 la 224,3 $\mathrm{J} \mathrm{kg}^{-1}$. Conform studiilor DSC anterioare, valorile mai mici ale $T_{\mathrm{d}}$ şi $\Delta_{\mathrm{d}} H$ reflectă scăderea stabilităţii termice a colagenului, cauzată de o deteriorare progresivă a acestuia [8].

\section{Proprietăţi TG}

Table 3: The percentage weight losses and $T_{\max }$ of aluminium tanned leather after artificial acid rain treatment

Tabelul 3: Pierderea în greutate în procente şi $T_{\max }$ pentru pielea tăbăcită cu aluminiu după tratamentul cu ploaie acidă artificială

\begin{tabular}{c|c|c|c|c|}
$\begin{array}{c}\text { Aging time (days) } \\
\text { Timp îmbătrânire } \\
\text { (zile) }\end{array}$ & $\begin{array}{c}\text { Weight loss in stage (1) (\%) } \\
\text { Pierderea în greutate în } \\
\text { faza (1) (\%) }\end{array}$ & $\begin{array}{c}\text { Weight loss in stage (2) (\%) } \\
\text { Pierderea în greutate în faza } \\
\text { (2) (\%) }\end{array}$ & $T_{\max }\left({ }^{\circ} \mathrm{C}\right)$ & $\Delta T_{\max }\left({ }^{\circ} \mathrm{C}\right)$ \\
\hline 0 & 5.6 & 48.0 & 324.1 & 0 \\
\hline 4 & 7.9 & 47.9 & 322.4 & 1.7 \\
\hline 8 & 8.8 & 52.1 & 316.9 & 7.2 \\
\hline 12 & 9.3 & 56.8 & 312.9 & 11.2 \\
\hline 16 & 10.0 & 59.3 & 312.3 & 11.8 \\
\hline
\end{tabular}


The pyrolysis process of aluminium tanned leather can be divided into two stages: stage (1) $50^{\circ} \mathrm{C} 150^{\circ} \mathrm{C}$ and stage (2) $260^{\circ} \mathrm{C}^{\sim} 600^{\circ} \mathrm{C}$. The percentage weight losses of two stages and the temperature of the maximum speed of the process obtained from TG and DTG curves were listed in Table 3.

As shown in Table 3, the percentage weight loss increases from $5.6 \%$ to $10 \%$ in the stage (1) and rises from $48.0 \%$ to $59.3 \%$ in the stage (2). The mass losses in the two stages increase gradually with the aging time of artificial acid rain which indicating the decreasing heat resistance of aluminium tanned leather. The values of the $T_{\max }$ (Table 3) decrease from 324.1 to $312.3^{\circ} \mathrm{C}$ and $\Delta T_{\max }$ is $11.8^{\circ} \mathrm{C}$ during the aging process. The modification of leather structure after artificial acid rain treatment can reduce the decomposition temperature and consequently reduce the thermal stability of leather [15].

The electrostatic attraction between aluminium tanning agent and collagen molecule is not firm, so the coordinate bonds were easily ruptured after the artificial acid rain treatment. Besides, the detanning and decrease of cross-linking degree may be occurred with the aging time. Therefore, the thermal stability and heat resistance of leather were reduced.

\section{Morphology and Structure}

SEM Analysis
Procesul de piroliză a pielii tăbăcite cu aluminiu poate fi împărţit în două etape: etapa (1) $50^{\circ} \mathrm{C} 150^{\circ} \mathrm{C}$ şi etapa (2) $260^{\circ} \mathrm{C}^{\sim} 600^{\circ} \mathrm{C}$. Procentele de pierdere în greutate în cele două etape şi temperatura vitezei maxime a procesului obţinute din curbele TG şi DTG au fost prezentate în Tabelul 3.

Aşa cum arată Tabelul 3, procentul de pierdere în greutate creşte de la 5,6\% la 10\% în etapa (1) şi creşte de la 48,0\% la 59,3\% în etapa (2). Pierderile de masă în cele două etape cresc treptat, odată cu timpul de îmbătrânire a pieilor tratate cu ploaie acidă artificială, ceea ce indică scăderea rezistenţei termice a pielii tăbăcite cu aluminiu. Valorile $T_{\max }$ (Tabelul 3) scad de la 324,1 la $312,3^{\circ} \mathrm{C}$, iar $\Delta T_{\max }$ este $11,8^{\circ} \mathrm{C}$ în timpul procesului de îmbătrânire. Modificarea structurii pielii după tratamentul cu ploaie acidă artificială poate reduce temperatura de descompunere şi, în consecinţă, stabilitatea termică a pielii [15].

Atracţia electrostatică dintre agentul tanant pe bază de aluminiu şi molecula de colagen nu este fermă, astfel încât legăturile coordinative s-au rupt uşor după tratamentul cu ploaie acidă artificială. În plus, detăbăcirea şi scăderea gradului de reticulare se pot produce odată cu creşterea timpului de îmbătrânire. Prin urmare, stabilitatea termică şi rezistenţa la căldură ale pielii au scăzut.

\section{Morfologie şi structură}

\section{Analiza SEM}
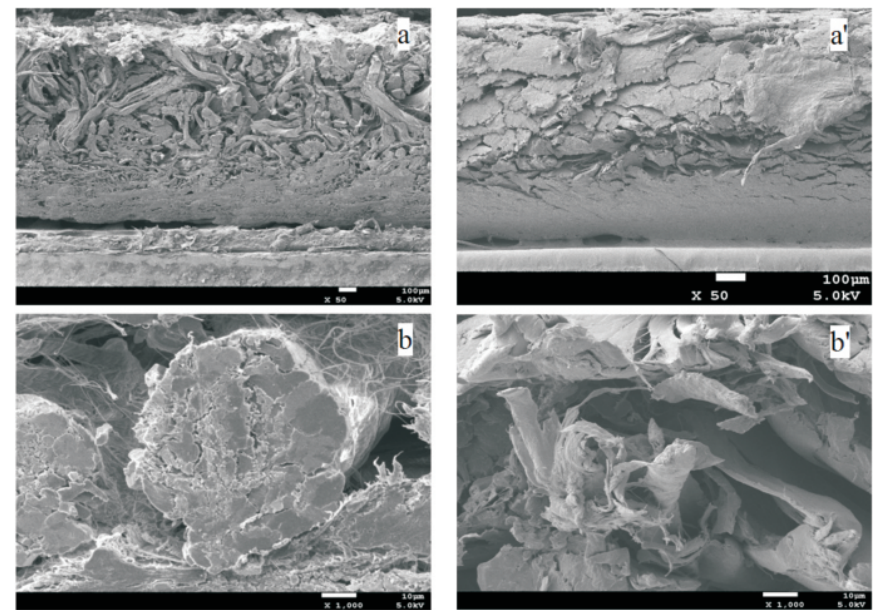

Figure 2. SEM micrographs of the cross sections of the matched sample and aged 16 days sample (a, a': 50x; b, b': 1000x)

Figura 2. Micrografii SEM ale probei iniţiale şi ale probei îmbătrânite după 16 zile în secţiune transversală (a, a': 50x; b, b': 1000x) 
The 50X SEM observation of the cross section after aging 16 days (Figure 2a') shows the collagen fibers are greatly damaged and the gaps among collagen fibres are disappeared compared with the matched samples (Fig. 2a). The random breakages of the fibre bundles are shown in the 1000X SEM micrograph (Fig. $2 b^{\prime}$ ) of aged 16 days compared with the matched samples (Fig. 2b). The fibre morphology was destroyed deeply by the artificial acid rain.

\section{FT-IR Analysis}

Observarea prin SEM la 50X a secţiunii transversale după 16 zile de îmbătrânire (Figura 2a') arată că fibrele de colagen sunt foarte deteriorate şi spaţiile dintre fibre au dispărut în comparaţie cu probele iniţiale (Figura 2a). Rupturile aleatorii ale fasciculelor de fibre sunt prezentate în microfotografia SEM la $1000 X$ (Figura 2b') a probei îmbătrânite după 16 zile, comparativ cu probele iniţiale (Figura 2b). Morfologia fibrelor a fost distrusă în profunzime de ploaia acidă artificială.

Table 4: The shift of Amide A, Amide I and Amide II bond of the matched sample and aged 16 days sample Table 4: Deplasarea benzilor amidă A, amidă I şi amidă II ale probei iniţiale şi ale probei îmbătrânite după 16 zile

\begin{tabular}{c|c|c|c|c|c|c}
$\begin{array}{c}\text { Aging time (days) } \\
\text { Timp imbătrânire } \\
\text { (zile) }\end{array}$ & $\begin{array}{c}\text { Amide A }\left(\mathrm{cm}^{-1}\right) \\
\text { Amida } A\left(\mathrm{~cm}^{-1}\right)\end{array}$ & $\begin{array}{c}\Delta \text { Amide A } \\
\Delta \text { Amida A }\end{array}$ & $\begin{array}{c}\text { Amide I }\left(\mathrm{cm}^{-1}\right) \\
\text { Amida I }\left(\mathrm{cm}^{-1}\right)\end{array}$ & $\begin{array}{c}\Delta \text { Amide I } \\
\Delta \text { Amida I }\end{array}$ & $\begin{array}{c}\text { Amide II }\left(\mathrm{cm}^{-1}\right) \\
\text { Amida II }\left(\mathrm{cm}^{-1}\right)\end{array}$ & $\begin{array}{c}\Delta \text { Amide II } \\
\Delta \text { Amida II }\end{array}$ \\
\hline 0 & 3300.00 & 0 & 1650.00 & 0 & 1550.00 & 0 \\
\hline 16 & 3424.59 & 124.59 & 1642.29 & -5.71 & 1541.51 & -8.49 \\
\hline
\end{tabular}

$\triangle$ Amide $\mathrm{A}=$ Amide $\mathrm{A}$ - the control; $\Delta$ Amide I = Amide I - the control; $\Delta$ Amide II = Amide II - the control $\triangle$ Amida $A=$ Amida $A$ - martor $\triangle \triangle$ Amida I = Amida I - martor; $\triangle$ Amida II = Amida II - martor

Table 4 represents the changes of amide $\mathrm{A}$ band $\left(3325-3330 \mathrm{~cm}^{-1}\right)$, amide I band $\left(1650-1665 \mathrm{~cm}^{-1}\right)$ and amide II band (1530-1550 $\left.\mathrm{cm}^{-1}\right)$ after aging 16 days. On one hand, the frequency of amide $A$ band is shifted to higher values greatly. On the other hand, the frequencies of amide I and amide II band are shifted to the range of lower values and the shift of amide II band is greater. The Amide A band moves from 3300.00 to $3424.59 \mathrm{~cm}^{-1}$ and $\Delta$ Amide I is $124.59 \mathrm{~cm}^{-1}$. The Amide I band shifts from 1648.66 to $1632.82 \mathrm{~cm}^{-1}$ and $\Delta$ Amide I is $15.84 \mathrm{~cm}^{-1}$. The Amide II moves from $1544.86 \mathrm{~cm}^{-1}$ to $1457.90 \mathrm{~cm}^{-1}$ and $\Delta$ Amide II is $86.96 \mathrm{~cm}^{-1}$. These results suggest that the role of acid rain destroys the ordered structure in collagen peptides and the main changes have been taken place on the group of $-\mathrm{CN}, \mathrm{C}=\mathrm{O}$ and $\mathrm{N}$ $H[16]$.

\section{CONCLUSIONS}

The aluminium tanned leather was aged with artificial acid rain and with the aging time, the thermal-stability and heat resistance were dropped
Tabelul 4 prezintă modificările benzilor amidă A $\left(3325-3330 \mathrm{~cm}^{-1}\right)$, amidă I $\left(1650-1665 \mathrm{~cm}^{-1}\right)$ şi amidă II (1530-1550 cm $\left.{ }^{-1}\right)$ după 16 zile de îmbătrânire. Pe de o parte, frecvenţa benzii amidă A este deplasată foarte mult la valori mai mari. Pe de altă parte, frecvenţele benzilor amidă I şi amidă II sunt deplasate în gama de valori mai mici, iar deplasarea benzii amidă II este mai mare. Banda amidă A se deplasează de la 3300,00 la $3424,59 \mathrm{~cm}^{-1}$ şi $\Delta$ amida I este la $124.59 \mathrm{~cm}^{-1}$. Banda amidă I se deplasează de la 1648,66 la 1632,82 cm $\mathrm{cm}^{-1}$ şi $\Delta$ amida l este la $15,84 \mathrm{~cm}^{-1}$. Amida II se deplasează de la $1544,86 \mathrm{~cm}^{-1}$ la $1457,90 \mathrm{~cm}^{-1}$ şi $\Delta$ amida II este la 86,96 $\mathrm{cm}^{-1}$. Aceste rezultate sugerează că ploaia acidă distruge structura ordonată a peptidelor din colagen şi principalele modificări au apărut la grupările $-\mathrm{CN}, \mathrm{C}=\mathrm{O}$ şi N-H [16].

\section{CONCLUZII}

Pielea tăbăcită cu aluminiu a fost îmbătrânită cu ploaie acidă artificială, iar stabilitatea termică şi rezistenţa la căldură au scăzut în mod clar odată cu 
obviously; the collagen fibers were damaged and the gaps of fibers were enlarged; amide $A$ band was moved to higher numbers, amide I and amide II band of collagen were gradually shifted to lower numbers. In conclusion, the artificial acid rain has a significant effect on aluminium tanned leather, leading morphology damaged and structure changed. Furthermore, acid air pollution has a great impact on the aluminium tanned leather historical relics and products.

\section{Acknowledgement}

This paper was written in the framework of Partnerships in Priority Areas-PNII Program conducted with the support of Romanian Ministry of Education \& UEFISCDI, project no. 325/2014, "Intelligent Strategy for Movable Cultural Heritage Monitoring in Changing Climate", INherit. creşterea timpului de îmbătrânire; fibrele de colagen sau deteriorat, iar spaţiile dintre fibre s-au extins; banda amidă A s-a deplasat la valori mai mari, benzile amidă I şi amidă II ale colagenului s-au deplasat treptat la valori mai mici. În concluzie, ploaia acidă artificială are un efect semnificativ asupra pielii tăbăcite cu aluminiu, ceea ce duce la deteriorarea morfologiei şi modificarea structurii. În plus, poluarea acidă a aerului are un impact semnificativ asupra relicvelor şi obiectelor istorice din piele tăbăcită cu aluminiu.

\section{Mulţumiri}

Această lucrare a fost realizată prin programul Parteneriate în domenii prioritare-PNII derulat cu sprijinul MEN \& UEFISCDI, proiect nr. 325/2014, "Intelligent Strategy for Movable Cultural Heritage Monitoring in Changing Climate", INherit.

\section{REFERENCES}

1. Doillon, C.J., Sliver, F.H., Biomaterials, 1986, 7, 3-7.

2. Xu-yong, L., Xin, L., Sichuan Leather, 2000, 23, 3, 50-52.

3. Deselnicu, V., Maier, S., Ioniuc, A., Geba, M., Deselnicu, D.C., Revista de Pielarie Incaltaminte (Leather and Footwear Journal), 2008, 8, 1, 3-11.

4. Krupińska, B., Worobiec, A., Gatto Rotondo, G., Novaković, V., Kontozova, V., Ro, C.U., Van Grieken, R., De Wael, K., Microchem J, 2012, 102, 49-53.

5. Deselnicu, V., Melniciuc, N., Maier, S., Albu, L., Deselnicu, D.C., Proceedings of ICAMS 2008, 23-24 October 2008, Bucharest, RO, 446-449.

6. Deselnicu, V., Melniciuc, N., Maier, S., Albu, L., Deselnicu, D.C., Proceedings of ICAMS 2008, 23-24 October 2008, Bucharest, RO, 449-452.

7. Waterer, J.W., Guide to the Conservation of Leather, Bull London, 1972.

8. Budrugeac, P., Badea, E., Della Gatta, G., Miu, L., Comanescu, A., Thermochim Acta, 2010, 500, 51-62.

9. Isik, N.O., Karavana, H.A., Revista de Pielarie Incaltaminte (Leather and Footwear Journal), 2010, 10, 4, 25-32.

10. Badea E., Carsote C., Vetter W., Petroviciu I., Miu L., Schreiner M., Budrugeac P., Della Gatta G., Proceedings of ICAMS 2012, 27-29 September 2012, Bucharest, 487-492.

11. Carsote, C., Miu, L., Petroviciu, I., Creanga, D.M., Giurginca, M., Vetter, W., Revista de Pielarie Incaltaminte (Leather and Footwear Journal), 2012, 12, 3, 183-192.

12. Xian-zhou, Li., The latest practical leather making process, Beijing, 1934.

13. Le-su, Y., Guang-yi, Z., Bin, Y., Ecologic Science, Aug 2005, 25, 254.

14. Larsen, R., J Soc Leath Tech Ch, 1993, 77, 151.

15. Marcilla, A., García, A.N., León, M., Martínez, P., Bańón, E., J Anal Appl Pyrol, 2011, 92, 194.

16. Petibois, C., Gouspillou, G., Wehbe, K., Delage, J.P., Deleris, G., Anal Bioanal Chem, 2006, 386, 1961-1966.

Article received/Data primirii articolului: 05.05.2015

Accepted/Acceptat la data: 11.06.2015 